\title{
Older Adults' Perceptions of Healthcare Overuse
}

\author{
Hyung J. Cho, $M D^{1,2}$, Michael Herscher, $M D^{7}$, and Akila Pai, $B S^{3}$ \\ 'Department of Medicine, Icahn School of Medicine at Mount Sinai, New York, NY, USA; ${ }^{2}$ Lown Institute, Brookline, MA, USA; ${ }^{3}$ Department of \\ Medical Education, Icahn School of Medicine at Mount Sinai, New York, NY, USA.
}

$\mathrm{J}$ Gen Intern Med 34(2):174

DOI: $10.1007 / \mathrm{s} 11606-018-4726-\mathrm{x}$

(c) Society of General Internal Medicine 2018

$\mathrm{L}$ etter to the editor

We applaud the recent findings by Green et al. and their recommendations regarding possible interventions to address patient perceptions of overuse. ${ }^{1}$ We agree that improving patient engagement by integrating shared decision making is a potential solution, but we suggest that an approach of tackling both overuse and underuse concurrently is needed as well.

The "less is more" movement has received recent criticism from clinicians as a one-sided, simplistic approach. ${ }^{2}$ Most would agree that less is not always more and that value is much more complex, and the public may have similar feelings of disillusionment. From the authors' findings, it is evident that even when asked specifically about overuse, patients voiced concerns of underuse. Particularly concerning is the jeopardy of trust with physicians, the belief that "doctors profit by keeping patients out of emergency departments." It is unsurprising that previous efforts to reduce overuse have been perceived as rationing by the public and media. ${ }^{3}$ Choosing Wisely has garnered impressive support from both clinician societies and consumer advocacy groups, such as Consumer Reports, in developing a collaborative approach to tackle overuse. Nevertheless, widespread awareness and implementation have been difficult to achieve, ${ }^{4,5}$ and patient engagement and understanding of overuse remain challenging.

In practice, both overuse and underuse of medical care services coexist from the singular patient visit to the workings of healthcare systems. In order to achieve "right care," an ethical and equitable approach to improving care for patients, both overuse and underuse need to be addressed. ${ }^{6}$ This can be done at the patient encounter where an elder patient who comes into the clinic with a recent fall after going to the bathroom can be evaluated for possible overtreatment with antihypertensives and other medications that may cause orthostasis. Concurrently, potential underuse of care should be addressed, such as assessing the need for a home safety evaluation or a grab bar in the bathroom. This concept of right care can also be applied at systems level, also. For this patient, a shared electronic medical record could help avoid repeating an echocardiogram that was recently done at another institution, while a standardized clinical pathway could ensure that appropriate testing, such as checking orthostatic blood pressures, is not overlooked. Taken together, this focus on both sides of the value coin - overuse and underuse - will help assuage both clinicians and patients and improve quality and value for our patients.

Corresponding Author: Akila Pai, BS; Department of Medical Education Icahn School of Medicine at Mount Sinai, One Gustave L Levy Pl, New York, NY 10029, USA (e-mail: akila.pai@icahn.mssm. edu).

\section{Compliance with Ethical Standards:}

Conflict of Interest: The authors declare no conflicts of interest.

\section{REFERENCES}

1. Green AR, Tung M, Segal JB. Older Adults' Perceptions of the Causes and Consequences of Healthcare Overuse: A Qualitative Study. J Gen Intern Med. https://doi.org/10.1007/s11606-017-4264-y

2. Rosenbaum L. The Less-Is-More Crusade - Are We Overmedicalizing or Oversimplifying?. N Engl J Med. 2017;377(24):2392-2397.

3. Campbell D. Doctors to withhold treatments in campaign against too much medicine'. The Guardian 2015 https://www.theguardian.com/ society/2015/may/12/doctors- to-withhold-treatments-in-campaignagainst-too-much-medicine.

4. Rosenberg A, Agiro A, Gottlieb M, et al. Early trends among seven recommendations from the Choosing Wisely campaign. JAMA Intern Med. 2015;175(12): 1913-1920.

5. Colla CH, Mainor AJ. Choosing Wisely Campaign: Valuable For Providers Who Knew About It, But Awareness Remained Constant, 2014-17. Health Affairs (Project Hope). 2017;36(11):2005-2011.

6. Saini V, Brownlee S, Elshaug AG, Glasziou P, Heath I. Addressing overuse and underuse around the world. Lancet. 2017. 